\title{
Three-Dimensional Finite Element Analysis for Stress Distribution During Intrusion and Retraction Using Orthodontic Mini-implants
}

\author{
Mohammed Abdul Naqeed ${ }^{1}$, Swaroopa Rani Ponnada ${ }^{2, ~ *, ~ V i v e k ~ R e d d y ~ G a n u g a p a n t a ~}{ }^{3}$ \\ ${ }^{1}$ Department of Orthodontics, Warangal, India \\ ${ }^{2}$ Department of Orthodontics, Panineeya Mahavidyalaya Institute of Dental Sciences and Research Centre, Hyderabad, India \\ ${ }^{3}$ Department of Orthodontics, Narayana Dental College, Nellore, India
}

\section{Email address:}

swaroopaponnada@gmail.com (S. R. Ponnada)

*Corresponding author

\section{To cite this article:}

Mohammed Abdul Naqeed, Swaroopa Rani Ponnada, Vivek Reddy Ganugapanta. Three-Dimensional Finite Element Analysis for Stress Distribution During Intrusion and Retraction Using Orthodontic Mini-implants. International Journal of Dental Medicine.

Vol. 7, No. 1, 2021, pp. 1-9. doi: 10.11648/j.ijdm.20210701.11

Received: December 8, 2020; Accepted: December 29, 2020; Published: April 26, 2020

\begin{abstract}
Background and Objectives: the study was conducted to evaluate the levels of stress produced in and around implants when placed at different sites for retraction and intrusion of maxillary anteriors. The other objective was to evaluate stress levels produced at implant bone interface when mini implants were placed at different angulations at the same site. Materials and Methodology: Finite element model of the maxilla and maxillary dentition was constructed using the CT skull of subject used in the study. All the accessories used like mini-implants, stainless steel wire, coil springs etc were scanned separately for obtaining detailed anatomic features. The force was given between retraction hook and mini -implant using NITI closed coil spring for intrusion and retraction of maxillary anterior teeth. The basic mechanical factors i.e. the stresses developed in mini implant, the angle of insertion of the mini implant into the bone surface were analyzed. Finite Element model was generated by Hyper mesh 9.0 software. Analysis was carried out by using Ansys 12.1 software. Results and Conclusion: All the set objectives were accomplished at the end of the study which included complete closure of extraction space by retraction and intrusion in the models. The following conclusions were drawn: The least stress was seen on an implant at middle third, when inserted at an angle of 800 to the bone surface, with application of 50-100mg of intrusive force on anterior implant and $200 \mathrm{gms}$ of retractive force on posterior implant.
\end{abstract}

Keywords: Hyper Mesh, Ansys, TADs, FEM, Retraction, Intrusion

\section{Introduction}

Anchorage as defined by T. M. Graber [1] is the nature and degree of resistance to displacement offered by an anatomic unit when used for the purpose of effecting tooth movement. Anchorage control plays a pivotal role in the effective management of orthodontic patients for obtaining structural and facial esthetics [2]. Depending on the need, Nanda [3] classified Anchorage as minimum, moderate or maximum anchorage.

As stated by the Newton's third law, every action has an equal and opposite reaction. Similarly, teeth during orthodontic treatment are subjected to forces and moments which always generate reciprocal forces of the same magnitude but opposite in direction. This is particularly relevant where such an action is favorable tooth movement and the reaction is unwanted tooth movement. To avoid these unwanted tooth movements, the reciprocal forces must be re-directed which can be achieved by reinforcing an orthodontic anchorage. [3]

Tooth-borne anchorage is one of the greatest limitations of modern orthodontics because teeth also tend to move in responses to forces during orthodontic treatment while extraoral anchorage can be used to supplement tooth borne anchorage and to deliver forces in direction not possible by intra-oral forces. Extra oral anchorage has severe limitations and requires patient cooperation. 
If skeletal anchorage could be applied to orthodontic tooth movement with readily removable implant anchored to jaws, forces might be applied to produce tooth movement in any direction without detrimental reciprocal forces [4].

The advent of micro-implants as skeletal anchorage devices has changed the scenario and has greatly expanded the boundaries of orthodontic tooth movement in all the three planes of space. The popularity of mini-implants is especially due to their ease of placement with less traumatic procedures, less patient discomfort, possibility of immediate loading, independent of patient compliance and high versatility 4 .

Finite element method can be applicable to study the problem of the stress-strain levels induced in internal structures. This method has potential for equivalent mathematical modeling of a real object of complicated shape and different materials [5].

Finite Element Method (FEM) was introduced as numerical analysis and it has become a useful technique for stress analysis in biologic system. The force system is currently the major factor that the orthodontist can control to achieve desirable orthodontic tooth movement generated from various appliances [6].

FEM offers an ideal method for accurate modeling of the tooth -periodontium system with its complicated three dimensional geometry. Force system that is used on orthodontic patient can be simulated; with FEM one can apply various force systems at any point and in any direction. The computer generates model images in which the different force levels are analyzed by color coding. [7]

In orthodontics, finite method studies have concentrated fundamentally on analysis of orthodontic appliances, dental movements and its relation to connective tissues. The two material properties essential for linear elastic FEM are Young's modulus and Poisson's ratio. Principal stresses are of 3 types- minor, intermediate and major depending on stress magnitude. Intermediate principal stresses depict areas of tension and compression. Stress magnitude was denoted by series of colors, in the spectrum of display. Green, blue, white, depicts progressively greater compression stress value. Darker pink, yellow, orange and red depicts greater magnitude of tension, light pink depicts no stress $[8,9]$.

\section{Aims and Objectives}

The aim of the study was to evaluate the stress levels associated with intrusion and retraction forces applied on maxillary anterior teeth of the created model with Temporary Anchorage Devices through FEM.

The objectives of the study was

1. To evaluate the stress levels produced in implants, when they are placed at different sites during retraction and intrusion of maxillary anteriors.

2. To evaluate stress levels produced at implant bone interface when implants are placed at different angulations at the same sites.

\section{Materials and Methods}

Finite element analysis was used for the study. After obtaining informed consent, Finite element model of the maxilla and maxillary dentition was constructed from CT scan of the skull of an individual visiting Department of Orthodontics, Mamata. Dental college, Kham am, Telangana, India. Implants, brackets, buccal tubes, NITI closed coil springs, 19x25 mil stainless steel wire were scanned separately for obtaining detailed anatomic features.

The finite element model of the maxillary dentition is created (Figure 1). It comprises of of maxillary dentition (except first premolars), brackets (MBT Prescription, 0.022, ORMCO) placed on teeth with 19X25 stainless steel arch wire with retraction hooks placed between lateral and canine brackets. Retraction force was given to anteriors by NITI closed coiled springs which were connected to implants and hooks. Implants were placed interdentally between the second premolar and first molar (on either side) and one in the anterior nasal spine.

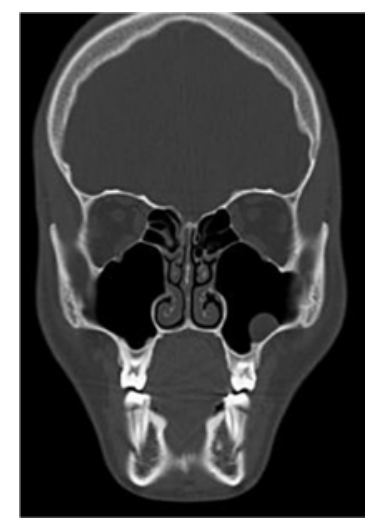

Figure 1. CT Scan Image of maxilla.

Three models were created for the study with different combinations of implants [10, 11].

Model I - one implant in anterior nasal spine region,

Model II - one implant in anterior nasal spine region, two implants one on each side interdentally between first molar and second premolar, (Figure 2)

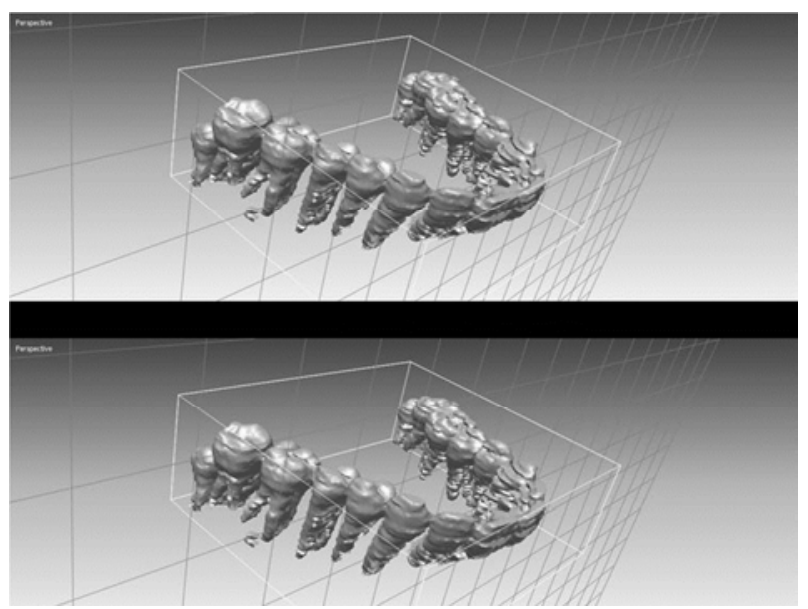

Figure 2. Maxillary teeth (Thresholding). 


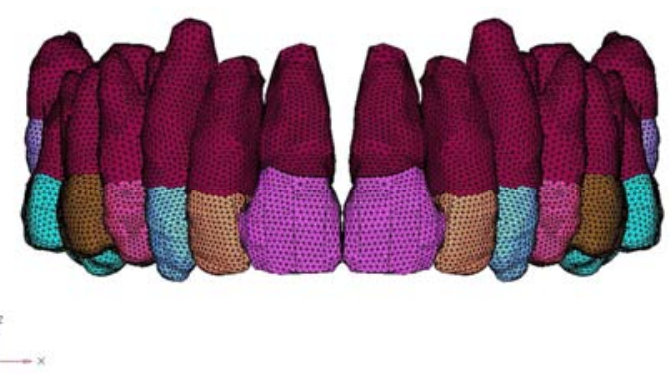

Figure 3. Geometric models of the maxillary teeth.

Model III - two implants one on each side interdentally between first molar and second premolar. (Figure 3)

Intrusion force of $100 \mathrm{Gms}$ and retraction force of $200 \mathrm{gms}$ was used and applied on the models. After application of load on implant, stresses in surrounding alveolar bone, and at implant bone interface were analyzed.

A. Hardware material details

B. Software details.

A. Hardware Material details $[9,10]$

Brackets- MBT. 022 versatile bracket prescription kit (ORMCO)

1. Implants- $1.4 \mathrm{mmx} 8 \mathrm{~mm}$ with insertion angulation of 80 degrees in anterior and 40 degrees in posterior region. (Denticon)

2. Stainless steel wire - 19x 25 stainless steel wires of 300 series alloys with high tensile strengths in excess of 300,000 psi (Ormco)

NITI Closed coil springs- Nickel titanium close spring of $12 \mathrm{~mm}$ in length (Orthotech). The passive length is $12 \mathrm{~mm}$ and when activated length increases to $24 \mathrm{~mm}$ producing $200 \mathrm{gm}$ of force.

B. Software details $[9,10]$

1. Computed Tomography machine (CT) - The CT scans acquire12-bit data (4,096 shades of gray) for each voxel

2. MIMICs 8.11 software - it allows to process and edit 2D image data (CT, $\mu \mathrm{CT}, \mathrm{MRI}$, etc.) to construct 3D models with the utmost accuracy, flexibility and userfriendliness. The powerful segmentation tools allow you to segment your medical CT/MRI images, take measurements and engineer directly on your 3D model. From there you can export your 3D data to a wide range of output formats and engineering applications; such as FEA, design, surgical simulation, additive manufacturing and more.

3. Solid edge 2004 software - Surface data of the tooth and implant, close-coil spring and maxilla is generated using this software.

4. Hyper mesh 9.0 software -Finite element model is generated using this software.

5. Ansys 12.1 software- The core product of Ansys Inc. is its ANSYS Multi-physics/ Structure mechanics module. This code is based on the Finite element method and is capable of performing static (stress) analysis, thermal analysis, model analysis, frequency response analysis, transient simulation and also coupled field analysis.

6. Others
Methodology

1. The geometric model of the each tooth and maxilla was obtained from the CT scan.

2. The geometric models of the implants, brackets, wire and close coil spring were modeled using Solid edge 2004 software by using reverse engineering technique (measuring the dimensions using precision tools)

3 . Both the geometric models (surface and line data) are then imported into Hyper mesh 9.0 software for meshing (the process of converting geometric model into finite element model is called meshing).

4. Finite element model consist of nodes and elements. Assembled finite element model of the maxilla, brackets, wire, spring and implants is then imported into Ansys software for analysis. Pre-processing, Solving and Post-Processing are three stages of analysis in Ansys 12.1 software.

5. The material properties (Young's modulus and Poisson's ratio) of the tooth, cortical bone, soft bone, PDL, implant, brackets, and wire are entered in the Preprocessing stage.

6. The loads and boundary conditions are applied in the solution stage.

A. Defining the Boundary Conditions-

The boundary conditions in FE models basically represent the load imposed on the structures under study and the area of the model which is restrained (in the present study the top cross-sectional area of the maxilla was restrained completely, linear and angular displacements were allowed).

B. Application of forces [12-14]

The following type of forces and moments were applied at the centre of slot of the brackets.

In the present study, forces were applied in horizontal and vertical direction for enmasse retraction and intrusion of anterior segment of maxilla. Intrusion force used was 100 Gms; retraction force used was $200 \mathrm{gm}$ which were applied on the models. After application of loads stresses on implant, stresses in surrounding alveolar bone, stresses at implant bone interface were analyzed.

C. Solving stage: solving each load case.

D. Post-processing:

The results are drawn and capturing the displacement and von-misses stress contours of each individual part of the system was done.

The result of the analysis is called 'post processing'. Stresses $(\mathrm{MPa})$ and the maximum displacements $(\mathrm{mm})$ were calculated and represented in colorful bands, different colors representing different stress levels and different values of displacements. Red color column of the spectrum indicates maximum principal stress following colors like orange, yellow, green, blue representing the reducing levels of stress and white color spectrum representing the lowest level of stress (Figure 4)

Minimum stress

Maximum stress. 


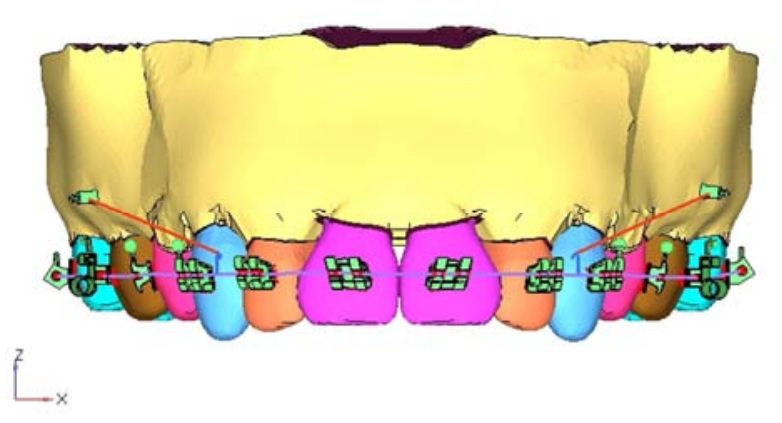

Figure 4. Geometric models of the maxillary teeth with brackets and maxillary bone (frontal view).

\section{Results}

With the post processing technique, results are obtained and represented with colored bands. Maximum stresses is indicated as "MX" and Minimum stresses is indicated as "MN

\section{VON-MISES STRESSES PRODUCED IN THE IMPLANT \\ Model I: (Figure 5)}

The following observations were made:

a) Maximum Von-Mises stresses on the implant is observed at the implant and bone interface in anterior region during intrusion (Red in color)

b) Minimum Von-Mises stresses on the implant is observed at the apex region (Blue in color)

c) The color band at the bottom indicates the stresses values at different level of the implant. for e.g.: The color dark yellow indicates the stress value $=0.6 \mathrm{MPa}$

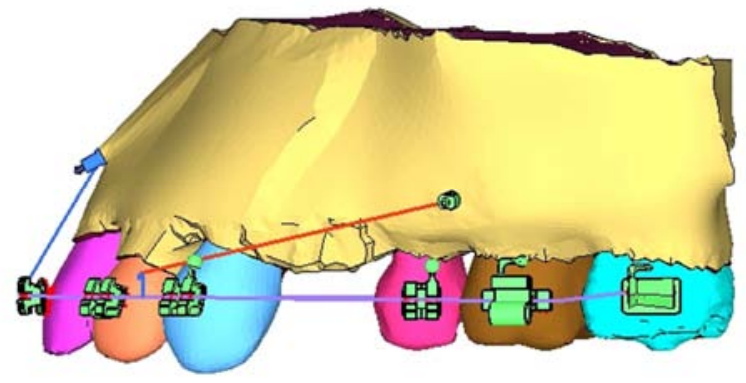

$L_{-Y}^{2}$

Figure 5. Geometric models of the maxillary teeth with brackets and maxillary bone (Lateral view).

\section{Model II: (Figure 6)}

a) Maximum vonMises stresses on the implant is observed at the neck region (light green in color)

b) A minimum vonMises stress on the implant is observed on the bottom most regions (Blue in color).

c) The color band at the bottom indicates the deformation values at different level of the implant and bone interface. For e.g.: The color dark green indicates the stresses value $=1.3 \mathrm{MPa}$.

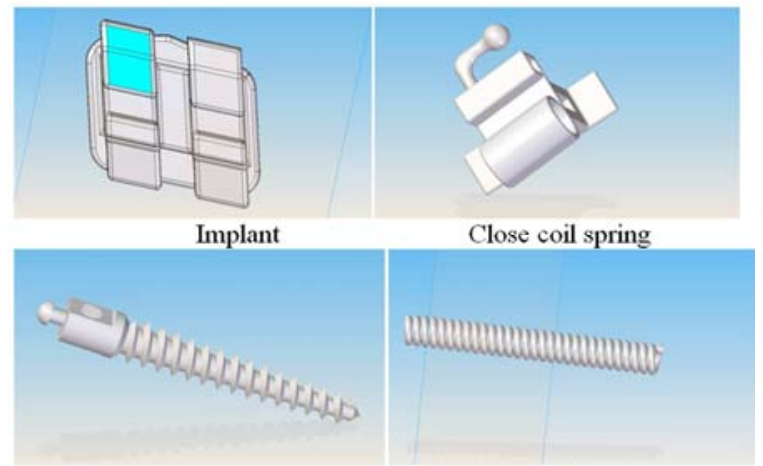

Figure 6. Geometric models of Brackets (ORMCO), Denticon Implants, NiTi Close coil springs (Orthtech). Geometricreconstruction of bracket Buccaltube maxillary 1st molar.

\subsection{Comparison Results of Above Two Models Indicates}

a) VonMises stresses on the neck of the anterior implant of model No: II were higher (1.3Mpa) when compared to model No: I (0.6Mpa).

b) The color band at the bottom indicates the deformation values at different level of the implant and bone interface. for e.g.: The color dark green indicates the stresses value $=2.6 \mathrm{MPa}$

Model No: III (Figure 7)

From above image the following observations are made:

a) Maximum vonMises on the implant is observed at the neck region in both implants during retraction (Red in color)

b) Minimum Von-misses of the implant is observed on the middle third to apex region (Blue in color)

c) The color band at the bottom indicates the deformation values at different level of the bone and implant for e.g. The color dark red indicates the vonMises stresses developed value $=3.2 \mathrm{MPa}$

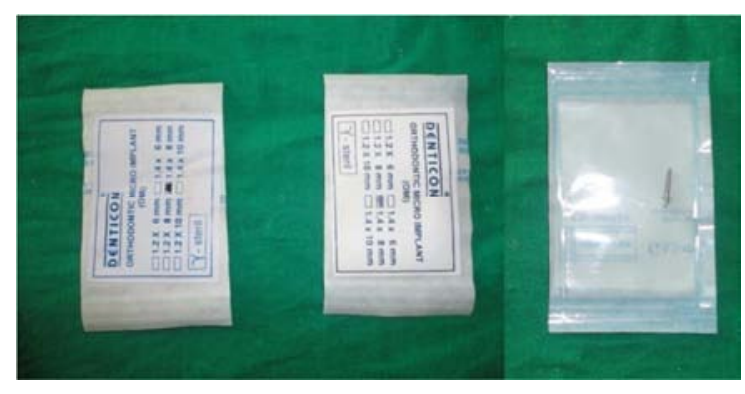

Figure 7. Denticon implant is $1.4 \mathrm{~mm}$ in diameter and $8 \mathrm{~mm}$ length.

\subsection{A Comparison Result of Above Two Models Indicates}

Von-Mises stresses on the neck of the posterior implant in model No: 3 (3.2Mpa) were higher, when compared to model No: 2 (2.6 MPa)

\section{STRESSES AT IMPLANT BONE INTERFACE}

Model No: I

The following observations were made:

a) Maximum von-Mises stresses on the implant is 
observed at the neck region (light green in color)

b) Minimum von-Mises stresses on the implant is observed on the bottom most region (Blue in color)

c) The color band at the bottom indicates the stresses values at different level of the implant and bone interface. for e.g.: The color dark green indicates the stresses value $=0.3-0.5 \mathrm{Mpa}$

Model No: II

The following observations were made: $h$

a) Maximum Von-Mises stresses on the implant is observed at the implant and bone interface in anterior region during intrusion (Red in color)

b) Minimum Von-Mises stresses on the implant is observed at the apex region (Blue in color)

c) The color band at the bottom indicates the stresses values at different level of the implant. for e.g.: The color dark yellow indicates the stress value $=(0.6$ $1.3 \mathrm{Mpa})$

Stresses at posterior implant bone interface:

Model No: III

The following observations were made:

a) Maximum Von-Mises stresses on the implant is observed at the neck region (Yellow in color)

b) Minimum Von-Mises stresses on the implant is observed on the bottom most region (Blue in color)

c) The color band at the bottom indicates the stresses values at different level of the implant and bone interface.for e.g.: The color dark green indicates the stress value $=(1.3-1.9 \mathrm{Mpa})$.

From above image the following observations are made:

a) Maximum vonMises stresses on the implant are observed at the neck region in both implants during retraction (Red in color)

b) Minimum vonMises stresses of the implant are observed on the middle third to apex region (Blue in color)

c) The color band at the bottom indicates the stresses values at different level of the bone and implant for e.g. The color dark red indicates the vonMises stresses developed value $=(0.6-1.9 \mathrm{Mpa})$.

\subsection{Comparison of the Above Three Models Result Shows}

Among all the implants used in these three models

Model No: 3 showed maximum amount of stresses at the neck of the implant.

\section{STRESSES AROUND SURROUNDING BONE \\ Model I: (Figure 8)}

The following observations were made:

a) Maximum vonMises stresses is observed at the anterior region on the maxillary hard bone (Yellow in color)

b) Minimum Von-misses stresses is observed on the posterior most region (Blue in color)

c) The color band at the bottom indicates the stresses values at different level of the bone for e.g.: The color green indicates the stress value. The Stresses produced in surrounding bone of anterior implant $=(0.2 \mathrm{Mpa})$

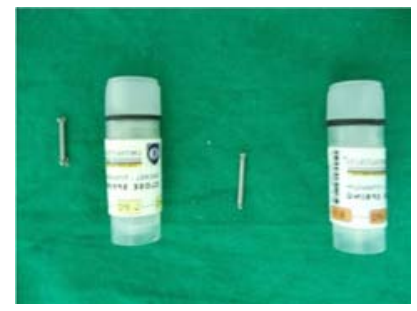

Figure 8. Orthotech Nickel- titanium close coil spring.

\section{Model II (Figure 9)}

The following observations were made:

a) Maximum Von-Mises stresses are observed at the posterior region on the maxillary hard bone (Yellow in color)

b) Minimum Von-Mises stresses are observed on the anterior most region (Blue in color)

c) The color band at the bottom indicates the stresses values at different level of the bone for e.g.: The color yellow indicates the stress value.

d) The stresses produced in surrounding bone of anterior implant $=0.0 \mathrm{Mpa}$

e) The stresses produced in surrounding bone of posterior implant $=0.6 \mathrm{MPa}$

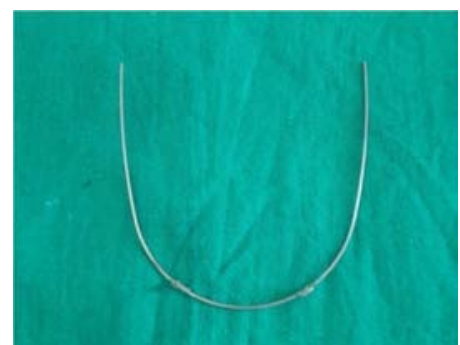

Figure 9. 19x25 Stainles steel (Ormco) arch wire with soldered buccal hooks in between lateral incisor and canine region.

Model III (Figure 10)

a) Maximum Von-Mises stresses were observed at the posterior region on the maxillary hard bone (Green in color)

b) Minimum Von-Mises stresses were observed on the anterior most region (Blue in color)

c) The color band at the bottom indicates the stresses values at different level of the bone for e.g.: The color yellow indicates the stress value.

d) The stresses produced in surrounding bone of posterior implant value $=0.6 \mathrm{Mpa}$

$$
\text { 1. Model No: I }
$$

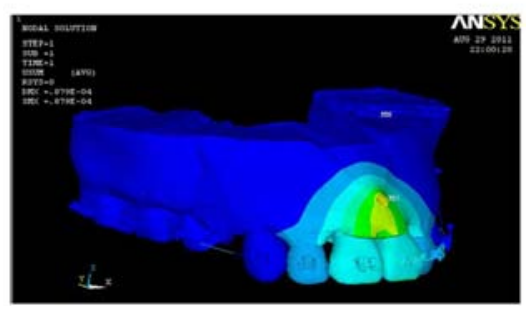

Figure 10. Antrioe single implant. 


\subsection{Comparison of Results of Above Shows}

a) Stresses in surrounding bone of anterior implant in Model No: II were less (0.0Mpa), when compared to model No: I (0.2Mpa).

b) Von-Mises stresses on the implant bone interface were high on the cervix and gradually decreased rapidly in the cortical bone.

c) Stresses in surrounding bone of posterior implant of model no: II (0.6 Mpa) and model no: III (0.6Mpa) were almost equal.

\section{Model No: II}

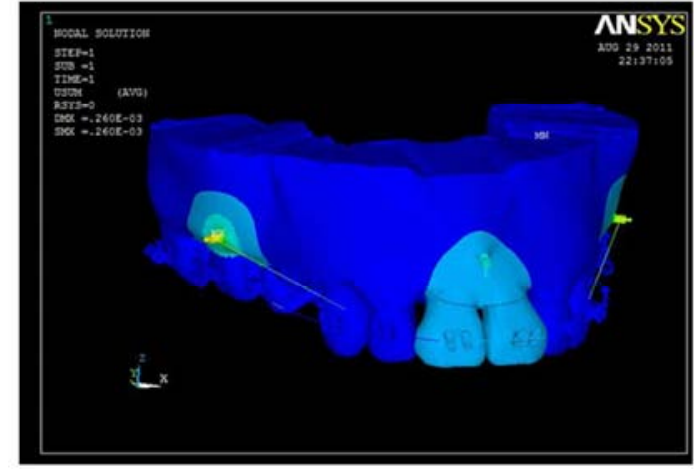

Figure 11. Anterior single and posterior two implants for intrusion and retraction.

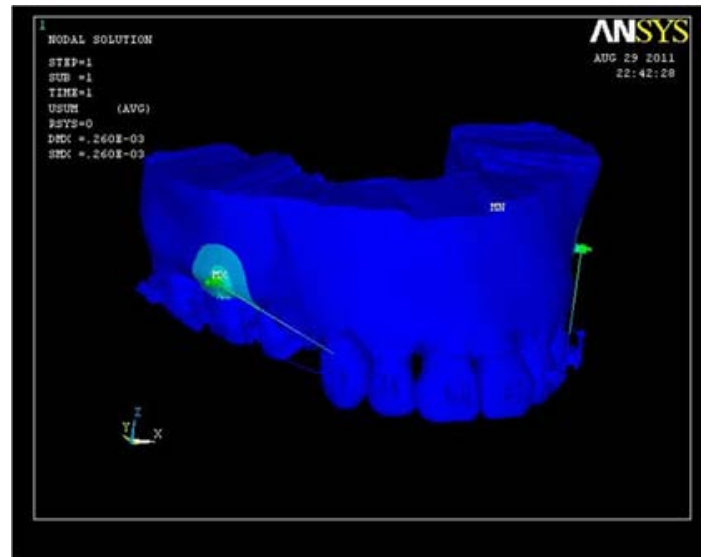

Figure 12. Two posterior implants for intrusion and retraction a. Stress in anterior implant.

\section{Model No: I}

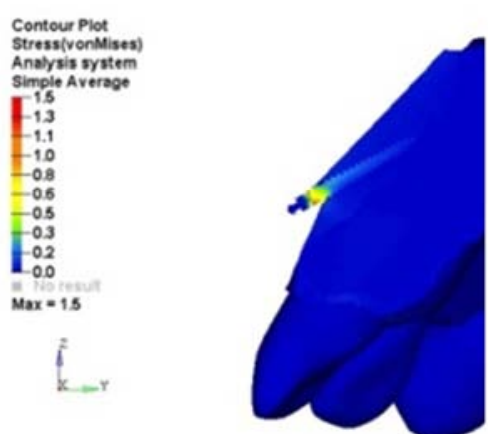

Figure 13. Stresses produced in anterior implant during intrusion.
Model 2
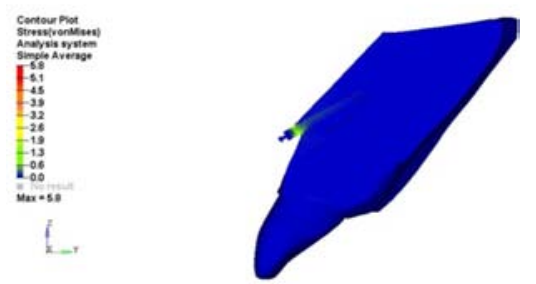

Figure 14. Stresses produced in anterior implant during intrusion.

Model No: II

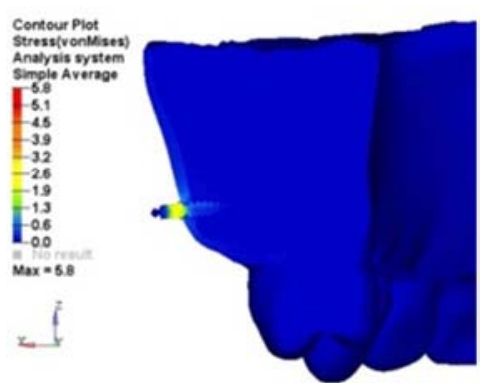

Figure 15. Stress in posterior implant during retraction and intrusion.

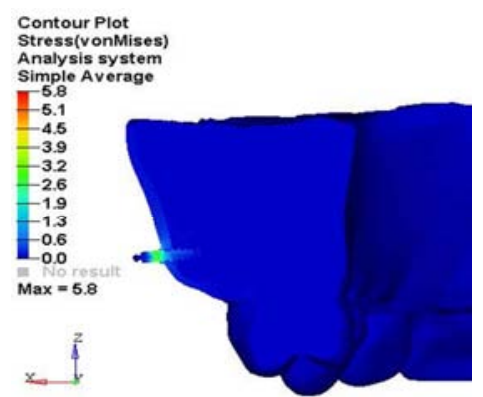

Figure 16. Stress in posterior implant during retraction and intrusion.

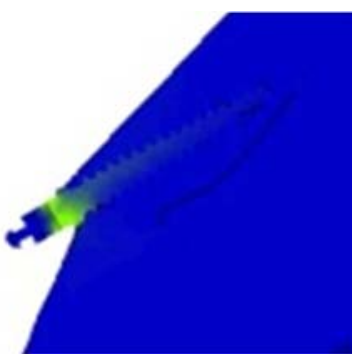

Figure 17. Stresses at Anterior implant implant bone interface during retraction.

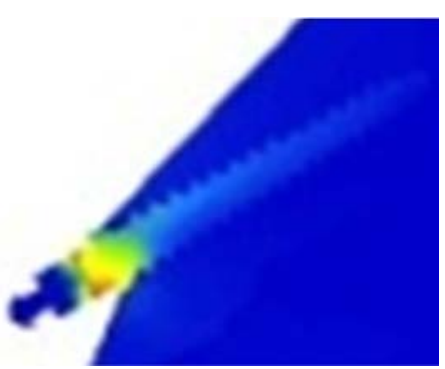

Figure 18. Stresses at Anterior implant implant bone interface during retraction. 


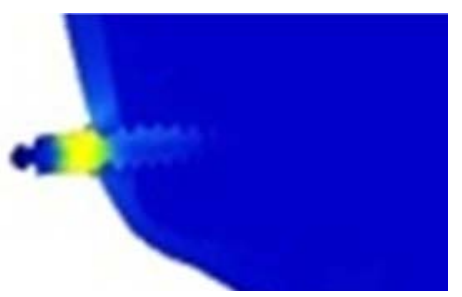

Figure 19. Stresses at Posterior implant bone interface during retraction and Intrusion.

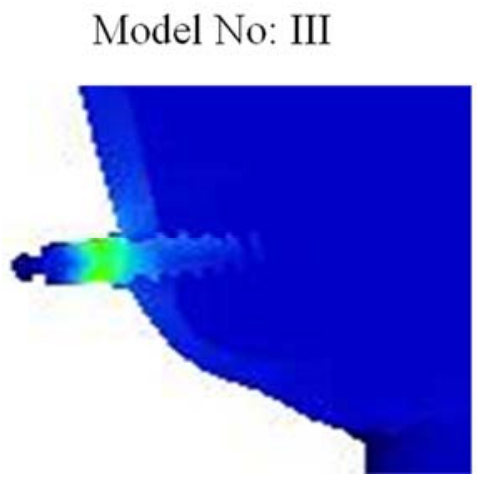

Figure 20. Stresses at Posterior implant bone interface during retraction and intrusion.

\section{Inference of the Results}

1. Von-Mises stresses on the neck of the anterior implant of model No: II were higher (1.3Mpa) when compared to model No: I ( $0.6 \mathrm{Mpa})$.

2. Von-Mises stresses on the neck of the posterior implant in model No: III (3.2Mpa) were higher, when compared to model No: II (2.6 MPa)

3. Among all the implants used in these three models, model No: III showed maximum amount of stresses at the neck of the implant.

4. Stresses in surrounding bone of anterior implant in model No: II were less $(0.0 \mathrm{Mpa})$, when compared to model No: I (0.2Mpa). \{insignificant difference\}

5. Stresses in surrounding bone of posterior implant of model no: II $(0.6 \mathrm{MPa})$ and model no: III $(0.6 \mathrm{Mpa})$ were almost equal.

6. Von-Mises stresses of the anterior implant at the bone interface in Model No: 1 (0.3- $0.5 \mathrm{Mpa})$ were lesser when compared to Model no: II (0.6-1.3Mpa)

7. Von-Mises stresses at the posterior implant bone interface of Model no: II (1.3-1.9Mpa) was less when compared to Model No: III (0.6-1.9Mpa).

8. Von-Mises stresses on the implant -bone interface were high on the cervix and gradually decreased rapidly in the cortical bone. Hence Von-Mises stresses at the implant bone interface were calculated at the cervix region for analyzing results.

\section{Discussion}

Anchorage considerations were major concerns for effective orthodontic treatment outcome. Poor anchorage control during treatment can results in loss of anchorage in all the three planes: Transverse (lingual rolling), Sagittal (mesialiasation), Vertical (extrusion). TADs play important role in maintaining absolute anchorage [13].

TADs are used for absolute anchorage where anchorage control of posterior segments during intrusion and retraction is very much needed. Mini implants either can be displaced or they can be fractured when using excess amount of forces applied. By studying the amount of stresses generated in and around the implant i.e.., stresses in the implant, stresses at implant-bone interface, stresses in surrounding bone, we can evaluate factors responsible for implant failures. The present study was done to evaluate stresses in and around implants during retraction and intrusion [14].

FEM analysis was selected for the study to analyse the stresses in and around the implant. Implants were placed posteriorly (interdentally between 2nd premolar and 1st molar) and anteriorly (below the anterior nasal spine) in the finite element model for effective retraction and intrusion of anteriors. In the study 40 degree for posterior implant and 80 degree for anterior implant were selected. Based on stress screen theory, within the implant-cortical bone and spongy bone system, the cortical bone received larger stress, while forces were conducted from micro-implant to the implant bone interface owing to the higher elastic modulus of cortical bone compared with that of spongy bone. Therefore implant with less tilted angle increases the contact area of microimplant and bone which in turn enhances the stability of the implant [15].

In present study 3 FEM models were used Model I implant placed in anterior region at 80 degree angulation and $90 \mathrm{gms}$ of intrusion force is applied.

In Model II - anterior implant is placed at 80 degree angulation with 90 gms of intrusion) force, posterior 2 implants at 40 degree angulation, retraction force of $200 \mathrm{gms}$ is applied and in Model III -posterior 2 implants (40degree angulation, retraction force of $200 \mathrm{gms}$ )

The present study evaluated the Von-Mises stress at the neck of the implant, at implant bone interface, and in the surrounding bone.

Highest concentrations of stresses were located at the neck of implants in all models which suggest the fracture probability will be higher at the neck of the implant when compare to other parts of the implant. This study is in accordance with Monica Vasquez et al [11] that highest stress concentration in the implant was localized in the cervical margin and the first screw $(1.2 \mathrm{~mm}$ apically from the cervical margin) between oseointergrated implant and its cortical bone, zero stress area in center of tooth. Hussein $\mathrm{H}$ et al [16]conducted a study and stated detailed stress distributions in the implant and peri-implant bone were found that Tightening loads caused much greater stress in the miniscrew and peri-implant bone. Over tightening a miniscrew after placement might be a more likely cause of fracture failure and bone trauma, the critical areas of stress in the loaded miniscrew were at the top 2 threads in the upper $2.5 \mathrm{~mm}$ of cortical bone. 
Single implant had more stress in the surrounding bone in anterior region it may lead to implant failure, which suggests that when two implants in anterior region are placed decreases stresses in alveolar bone. A study by JinHwa Lee ET al [17] says when mini-implants fail during orthodontic treatment, there is a need to either replace the failed implant in the adjacent interradicular area or wait for the bone to heal before replacing the mini-implant. When mini-implants loosen during active orthodontic treatment, replace them at adjacent interradicular spaces with better alveolar bone mass. A study by Joseph S. Petreya [18], significantly greater force to fail was required for a 909degree insertion angle than for 45 or 135 degree insertion angles. No significant difference was found between the 45 and 135 degree insertion angles. A significant reduction in force to fail occurred when comparing 90 and 45 degree oblique insertion angles. Placement of TADs at 90 degree to the cortical plate is the most retentive insertion angle. Insertion at an oblique angle from the line of force reduces retention of TAD. Telma Martins [19] et al says that one mini-implant should be placed as low as possible between the centrals. In this position, the force line will extend across the front of the set's resistance center, thereby generating an intrusion effect.

Biomechanically the point of force application for intrusion in anterior segment should be between lateral incisors and canines $(3 \mathrm{~mm}-4 \mathrm{~mm}$ from the CEJ). A Study by Madhur Upadhyay et al [20] showed segmental intrusion of only the maxillary incisors using skeletal anchorage by placing a mini-implant between the central incisors below the ANS. mini-implant anchorage was used to intrude the maxillary anterior teeth en masse by $4 \mathrm{~mm}$ using a segmented arch wire. selection of the point of application of the intrusive force with respect to the centre of resistance (Cres) of the anterior segment. The Center of resistance of the six anterior teeth was estimated to be halfway between the Cres of the four incisors and canines. True intrusion without axial inclination change can only be obtained by directing the intrusive force through the center of resistance of the anterior teeth. A study by Sang-Jin Sung ET al [21] the mini-implant was positioned approximately three $\mathrm{mm}$ above the root apex. A light force $(20 \mathrm{~g})$ was maintained until the mini-implant was removed 15 months late. The overbite was corrected from $7.2 \mathrm{~mm}$ to $1.7 \mathrm{~mm}$ by upper incisor intrusion.

Vertical control will be less if we place only two implant in posterior region (model no: 3), So one implant should be placed anteriorly and two in posterior region, as shown in Model; 2 to have an adequate control during intrusion and retraction of anterior maxillary segment. a study by Madhur Upadhyay et al [20] mini-implants placed between the roots of the second premolar and first molar. implants were loaded with pre-calibrated nickel-titanium closed coil spring (150) extending from implant head to the crimpable hooks to $0.017 \times 0.025$ stainless steel arch wire for enmasse retraction of the anterior teeth it provides effective control over the posterior dentoalveolar dimension with dramatic improvement in facial esthetics.

In present study, stresses in surrounding bone and implant bone interface were negligeable in all models except anterior model no: 1 with single implant. A study by Park Hs et al13 was done to find the effect of insertion angle on the stability of mini implants. In this study they analyzed the effects of all the insertion angles from that are 300 to 900 with a range of 100. They found that the angle of insertion had a significant effect on primary stability of mini implant. The best primary stability was achieved by an insertion angle ranging from 600 to 700 , a more oblique direction of insertion seems to be favorable to minimize the risk of root contact.

In my study Von-Mises stresses on the implant bone interface were high on the cervix and gradually decreased rapidly in the cortical bone. Martin I. Puente et al [8]analysed the distribution of the stress on dental and periodontal structures. Von-Mises stress were determined at the root, alveolar bone and periodontal ligament (PDL). The dental apex and bony alveolar crest zones are the areas that suffer the greatest stress.

According to Vasquez Metal [21] Highest stresses occurred in the implant followed by the root surface, cortical bone and periodontal ligament.

Among all the implants used in these three models, model No: III showed maximum amount of stresses at the neck of the implant. Gallas MM et al [22] results showed that the area with the highest stress around the dental implant when used as orthodontic anchorage and the surrounding bone was the cervical margin. This finding is clinically important in order to preserve the bone-implant interface in this area. [23]

\section{Conclusions}

1. Von-Mises stresses on the neck of the anterior implant of model No: II were higher (1.3Mpa) when compared to model No: I (0.6Mpa).

2. Von-Mises stresses on the neck of the posterior implant in model No: III (3.2Mpa) were higher, when compared to model No: II (2.6 Mpa)

3. Among all the implants used in these three models, model No: III showed maximum amount of stresses at the neck of the implant.

4. Stresses in surrounding bone of anterior implant in model No: II were less $(0.0 \mathrm{Mpa})$, when compared to model No: I (0.2Mpa). \{insignificant difference\}

5. Stresses in surrounding bone of posterior implant of model no: II (0.6 MPa) and model no: III (0.6Mpa) were almost equal.

6. Von-Mises stresses of the anterior implant at the bone interface in Model No: $1(0.3-0.5 \mathrm{Mpa})$ were lesser when compared to Model no: II (0.6-1.3Mpa)

7. Von-Mises stresses at the posterior implant bone interface of Model no: II (1.3-1.9Mpa). was less, when compared to Model No: III (0.6-1.9Mpa).

8. Von-Mises stresses on the implant-bone interface were high on the cervix and gradually decreased rapidly in the cortical bone. 


\section{References}

[1] Lindquist JT. The edgewise appliance. In: Graber TM, editor. Orthodontics: current principles and techniques. St Louis: Mosby; 1985: 565-640.

[2] Proffit WR. Biomechanics and mechanics. In: Profit WR, Fields HW Jr, editors. Contemporary orthodontics. St Louis: Mosby, 2000: 295-362.

[3] Nanda R, Kuhlberg A. Biomechanical basis of extraction space closure. In: Nanda $R$, Kuhlberg A, editors. Biomechanics in clinical orthodontics. Philadelphia: W. B. Saunders; 1996. P. 156-87.

[4] Creekmore TD, Eklund MK. The possibility of skeletal anchorage. J Clin Orthod 1983; 17: 266-269.

[5] Fotos PG, Spyrakos CC, Bernard DO. Orthodontic forces generated by a simulated arch wire appliance evaluated by the finite element method. Angle Orthod1987; 60 (4): 277-282.

[6] Tanne K, Koenig HA, Burstone CJ. Moment to force ratios and center of rotation. Am J Orthod Dentofac Orthop 1988; 94 : 426-34.

[7] Cobo J, Sicilia A, Argüelles J, Suárez D, Vijande M. Initial stress induced in periodontal tissue with diverse degrees of bone loss by an orthodontic force: tridimensional analysis by means of the finite element method. Am J Orthod Dentofacial Orthop. 1993 Nov; 104 (5): 448-54.

[8] Puente MI, Galban L, Cobo JM. Initial stress differences between tipping and torque movements. A three dimensional finite element analysis. Eur. J. Orthod, 18 (1996) 329-339.

[9] Katona TR. A comparison of the stresses developed in tension, shear peel, and torsion strength testing of direct bonded orthodontic brackets. Am J Orthod Dentofacial Orthop. 1997 Sep; 112 (3): 244-51.

[10] Tanne K, Yoshida S, Kawata T, Sasaki A, Knox J, Jones ML. An evaluation of the biomechanical response of the tooth and periodontium to orthodontic forces in adolescent and adult subjects. Br J Orthod. 1998 May; 25 (2): 109-15.

[11] Vasquez M, Calao E, Becerra F, Ossa J, Enriquez C, Fresneda E. Initial stress differences between sliding and sectional mechanics with an Endosseous implant as anchorage: A 3dimensional finite element analysis. Angle Orthod2001; 71 (4): 247- 256 .

[12] Jones ML, Hickman J, Middleton J, Knox J, Volp C. A validated finite element method study of orthodontic tooth movement in the human subject. J Orthod. 2001 Mar; 28 (1): 29-38.
[13] Park HS, Bae SM, Kyung HM, Sung JH. Micro-implant anchorage for treatment of skeletal class I bialveolar protrusion. J Clin Orthod 2001; 35: 417422.

[14] H.-J. Chun, S.-Y. Cheong, J.-H. Han, S.-J. Heo, J.-P. Chung, I.-C. Rhyu, Y.-C. Choi, H.-K. Baik, Y. Ku, M.-H. Kim. Evaluation of design parameters of osseointegrated dental implants using finite element analysis. Journal of Oral Rehabilitation 2002 June; 29: 565-574.

[15] Geramy A. Initial stress produced in the periodontal membrane by orthodontic loads in the presence of varying loss of alveolar bone: a three-dimensional finite element analysis. Eur J Orthod. 2002 Feb; 24 (1): 21-33.

[16] Hussein H. Ammar, Peter Ngan, Richard J. Crout, c Victor H. Mucino, and Osama M. Mukdadid. Three-dimensional modeling and finite element analysis in treatment planning for orthodontic tooth movement. Am J Orthod Dentofacial Orthop 2011; 139: e59-e71.

[17] Jin-Haw Lee, HyeRan Choo, Seong-Hun Kim, Kyu-Rhim Chung, Lucille A. Giannuzzi, and Peter Ngan. Replacing a failed mini-implant with a miniplate to prevent interruption during orthodontic Treatment Am J Orthod Dentofacial Orthop 2011; 139: 849-57 June.

[18] Joseph S. Petreya; Marnie M. Saundersb; G. Thomas Kluemperc, Larry L. Cunninghamd, Cynthia S. Beemane. Temporary anchorage device insertion variables: effects on retention. Angle Orthodontist, Vol 80, No 4, 2010.

[19] Telma Martins de Araújo, Mauro Henrique Andrade Nascimento, Fernanda Catharino Menezes Franco, Marcos Alan Vieira Bittencourt.: Dental Press J. Orthod. 3 v. 13, no. 5, p. 36-48, Sep./Oct. 2008.

[20] Madhur Upadhyay, K. Nagaraj, Sumit Yadav, Ruchi Saxena Mini-implants for en masse intrusion of maxillary anterior teeth in a severe Class II division 2 malocclusion. Journal of Orthodontics, Vol. 35, 2008, 79-89.

[21] Sang-Jin Sung, Gang-Won Jang, Youn-Sic Chun, and YoonShik Moon. Effective enmasse retraction design with orthodontic mini-implant anchorage: A finite element analysis. Am J Orthodox Dentofacial Orthop 2010; 137: 648-57.

[22] Gallas MM, Abeleira MT, Fernandez JR, Burguera M. Threedimensional numerical simulation of dental implants as orthodontic anchorage. Eur J Orthod 2005; 27: 12-16.

[23] HedayatiZ, Shomali M. Maxillary anterior en masse retraction using different antero-posterior position of mini screw: a 3D finite element study. Prog Orthod 2016; 17: 31-35. 